\title{
A Convolution-based DCT Algorithm
}

\author{
Yi-. Wing Chin and Ja-Ling Wu \\ Department of Computer Science and Information Engineering \\ National Taiwan University
}

\begin{abstract}
In this paper, based on some theorems of Number theory, a new convolution-based algorithm for computing the DCT (with power of two length) is proposed. In terms of computational counts, the proposed algorithm computes a length- $\mathrm{N}$ DCT (with $\mathrm{N}$ a power of two) using only $\mathrm{N}$ multiplications.
\end{abstract}

\section{Introduction}

Since the discovery of the discrete cosine transform (DCT) [1] , many new algorithms for computing the DCT have been developed. These algorithms are either indirect computations using fast Fourier transforms [1]-[2] or direct computations using matrix factorization (or recurisive computation) [3] . On the other hand,the convolution-based approach deals commonly with the prime length (Prime factors) DFTs [4] . These algorithms can be optimized using the Winograd's convolution algorithm [5], or be implemented using the number theoretical transform (NTT) which needs only order $\mathrm{N}$ multiplications.

In this paper, based on some theorems of Number theory, a new convolution-based algorithm for computing the DCT (with power of two length) is proposed. In terms of computational counts, the proposed algorithm computes a length-N DCT (with N a power of two) using only N multiplications.

2 Some Useful Theorems in Number Theory and the Properties of DCT
Theorem 1 If $n>2$, then

$$
\begin{aligned}
& 4 k+1 \equiv 5^{\beta_{1}} \quad\left(\bmod 2^{n}\right) \\
& 4 k+3 \equiv-5^{\beta_{2}} \quad\left(\bmod 2^{n}\right)
\end{aligned}
$$

where $k \in Z$ (the set of integers) and $\beta_{1}, \beta_{2} \in Z^{+}$(the set of positive integers).

Theorem 2 If $n>2$, then

$$
5^{2^{n-3}} \equiv 1+2^{n-1} \quad\left(\bmod 2^{n}\right)
$$

The proofs of Theorem 1 and 2 can be found in [6]

Theorem 1 implies that there is a one-to-one mapping between the following two subsets in $Z_{2^{n}}$ (the integers modulo $2^{n}$ ) that is

$\left\{4 t+1 \mid t=0,1, \cdots, 2^{n-2}-1\right\} \longleftrightarrow\left\{5^{t} \mid t=0,1, \cdots, 2^{n-2}-1\right\}$.

Corollary 1 For the matrix of index functions

$$
M=[f((4 i+1)(4 j+1) \quad(\bmod 4 N))]_{i, j=0.1, \cdots, N-1}
$$

there exist a circular convolution matrix $C$ and two permutation matrix $P_{1}$ and $P_{2}$, such that

$$
M=P_{1} C P_{2}
$$

[proof]: By theorem 1

$$
4 i+1 \equiv 5^{t} \quad(\bmod 4 N)
$$

Therefore, we can reorder the rows and columns in $M$ i.e.,

$$
\begin{aligned}
C & =\left[\begin{array}{ll}
f\left(\left(5^{N-t_{1}} \cdot 5^{t_{2}}\right)\right. & (\bmod 4 N)
\end{array}\right] \\
& =\left[\begin{array}{ll}
f\left(5^{t_{2}-t_{1}}\right. & (\bmod 4 N))
\end{array}\right]_{t_{1}, t_{2}=0,1, \cdots, N-1}
\end{aligned}
$$


Thus, $C$ is a circular convolution matrix, and the input and output reordering processes can be achieved by two permutation matrices ( say $P_{1}$ and $P_{2}$ ), respectively.

According to Wang [3], there are four types of DCT definitions and the computation of the four types of DCT can be reduced to the computation of the type-IV DCT. Therefore, the fast algorithms for any type of the DCT depends only on the computation of type-IV DCT .

\section{Proposed Algorithm for Computing Type-IV DCT}

From [3] , the type-IV DCT can be rewritten as

$$
X(k)=\sum_{n=0}^{N-1} x(n) \cdot \cos \left(\frac{2 \pi(2 n+1)(2 k+1)}{8 N}\right)_{k=0,1, \cdots, N-1}
$$

We will prove that the work for computing N-point typeIV DCT can be achieved by computing an N-point skew circular convolution and permutations using the following processes.

STEP 1. Extend $\left[C_{N}^{I V}\right]$ (the notations defined in [3] are adopted in the rest of this paper for simplicity) as follows:

$$
Y(k)=\sum_{n=0}^{2 N-1} y(n) \cdot \cos \left(\frac{2 \pi(2 n+1)(2 k+1)}{8 N}\right)_{k=0,1, \cdots, 2 N-1}
$$

where

$$
y(n)=\left\{\begin{array}{cl}
x(n) & 0 \leq n \leq N-1 \\
0 & N \leq n \leq 2 N-1
\end{array}\right.
$$

and then

$$
X(k)=Y(k) \quad \text { for } k=0,1, \cdots, N-1
$$

STEP 2. Reorder the input and output sequence.

Similar to the previous work [2] the above $2 \mathrm{~N}$-point transform can be rewritten as :

$$
\bar{Y}(k)=\sum_{n=0}^{2 N-1} \tilde{y}(n) \cos \left(\frac{2 \pi(4 n+1)(4 k+1)}{8 N}\right) \quad k=0,1, \cdots, 2 N-1
$$$$
\text { where }
$$$$
\left\{\begin{array}{ll}
\tilde{y}(n) & =y(2 n) \\
\tilde{y}(2 N-n-1) & =y(2 n+1)
\end{array} \quad n=0,1, \cdots, N-1\right.
$$

and

$$
\left\{\begin{array}{ll}
\tilde{Y}(k) & =Y(2 k) \\
\tilde{Y}(2 N-k-1) & =Y(2 k+1)
\end{array} \quad k=0,1, \cdots, N-1\right.
$$

STEP 3. The matrix representation of $(2)$ is

$$
G_{2 N}=\left[\cos \left(\frac{2 \pi(4 n+1)(4 k+1)}{8 N}\right)\right]_{n, k=0,1, \cdots, 2 N-1}
$$

From corollary 1 , it follows that the following equation hold :

$$
G_{2 N}=P_{2 N} C_{2 N} Q_{2 N}
$$

where $P_{2 N}$ and $Q_{2 N}$ are two permutation matrices and $C_{2 N}$ is a $2 \mathrm{~N}$-point circular convolution matrix and can be represented as :

$$
C_{2 N}=\left[\cos \left(\frac{2 \pi \cdot 5^{(j-i)}}{8 N}\right)\right]_{i, j=0.1, \cdots, 2 N-1}
$$

STEP 4. Since

$$
\cos \left(\frac{2 \pi \cdot 5^{n+N}}{8 N}\right)=-\cos \left(\frac{2 \pi \cdot 5^{n}}{8 N}\right)
$$

(by theorem 2 , details are in the Appendix)

$$
\begin{aligned}
C_{2 N} & =\left[\begin{array}{rr}
H_{N} & -H_{N} \\
-H_{N} & H_{N}
\end{array}\right] \\
& =\left[\begin{array}{r}
I_{N} \\
-I_{N}
\end{array}\right] \cdot\left[H_{N}\right] \cdot\left[\begin{array}{ll}
I_{N} & -I_{N}
\end{array}\right]
\end{aligned}
$$

where $H_{N}$ is the so-called N-point skew circular convolution matrix.

By (4), it follows that the computation of $C_{2 N}$ can be achieved by calculating an $\mathrm{N}$-point skew circular convolution and additional $\mathrm{N}$ additions/substractions.

Remark 1: In step 1,we extend the input sequence with $\mathrm{N}$ zeros, therefore, the $\mathrm{N}$ additions/substractions in step 4 can be replaced by the "sign changen operations.

Remark 2: In step 1, we only need half of the output sequence. Therefore, the post-operations of (4) can be achieved by "sign change" operations.

According to the above discussion, we can conclude that the computation of an N-point type-IV DCT can be achieved by an N-point skew circular convolution with some permutations and sign changes of in put and output sequences. From [7] , an N-point skew circular convolution (or the polynomial procudt modulo $z^{n}+1$ ) can be computed by means of the generalized number theoretical transform (GNTT) with only $\mathrm{N}$ multiplications.

\section{Algorithms for Discrete Sinusoidal}

\section{Transforms}

According to the previous works $[3,8,9]$, the relations between some well-known discrete sinusoidal transforms ( DFT , DHT (discrete Hartley transform), DCT and DST (discrete Sine transform) ) are very clear, and listing as follows :

$$
\begin{aligned}
D F T(N) & \Rightarrow\left\{\begin{array}{l}
D F T\left(\frac{N}{2}\right) \\
t w o D C T^{I I}\left(\frac{N}{4}\right)
\end{array}\right. \\
D H T(N) & \Rightarrow\left\{\begin{array}{l}
D H T\left(\frac{N}{2}\right) \\
t w o D C T^{I I}\left(\frac{N}{4}\right)
\end{array}\right.
\end{aligned}
$$




$$
\begin{aligned}
& D C T^{I I}(N) \Longrightarrow\left\{\begin{array}{l}
D C T^{I I}\left(\frac{N}{2}\right) \\
D C T^{I V}\left(\frac{N}{2}\right)
\end{array}\right. \\
& D S T^{I I}(N) \Longrightarrow\left\{\begin{array}{l}
D S T^{I I}\left(\frac{N}{2}\right) \\
D C T^{I V}\left(\frac{N}{2}\right)
\end{array}\right.
\end{aligned}
$$

Based on the discussion of section 3, we can compute the $D C T^{I V}(N)$ using N-point skew circular convolution $(S C C(N))$. Therefore, the following result can be derived by the recursive formulas (5)-(8).

$$
\begin{aligned}
& D F T(N) \quad \Longrightarrow \operatorname{two} \operatorname{SCC}\left(\frac{N}{4}\right), t w o \operatorname{SCC}\left(\frac{N}{8}\right), \cdots \\
& D H T(N) \Longrightarrow \operatorname{two} \operatorname{SCC}\left(\frac{N}{4}\right), t w o \operatorname{SCC}\left(\frac{N}{8}\right), \cdots \\
& D C T^{I I}(N) \Longrightarrow \operatorname{SCC}\left(\frac{N}{2}\right), \operatorname{SCC}\left(\frac{N}{4}\right), \cdots \\
& D_{S T^{I I}(N)} \Longrightarrow \operatorname{SCC}\left(\frac{N}{2}\right), \operatorname{SCC}\left(\frac{N}{4}\right), \cdots
\end{aligned}
$$

with some interblock additions and sign changes.

Remark 3: Although the DFT is defined in the complex number system, we can still derive an algorithm using only real SCCs.

\section{Conclusion}

In this paper,we have developed an algorithm which transfers the problem of N-point type-IV DCT into the problem of N-point skew circular convolution.In theory, this algorithm can achieve the lower bound of the number of multiplications according to the minimum complexity polynomial algorithms. In practice, by means of the number theoretical transform,we can compute $\left[C_{N}^{I V}\right]$ using only $\mathrm{N}$ multiplications, or we can use a filter-type structure that is very suitable for the VLSI implementation.

According to the relations between type-IV DCT and other famous transforms, we have mentioned that the other discrete sinusoidal transforms can be computed by means of the combination of some SCCs of smaller size, and possess the same advantages in both theoretical and practical as the type-IV DCT.

\section{Appendix}

[proof of eq. 3]:

$$
\begin{aligned}
& \cos \left(\frac{2 \pi \cdot 5^{(n+N)}}{8 N}\right) \\
= & \cos \left(\frac{2 \pi \cdot 5^{n} \cdot(1+4 N)}{8 N}\right) \quad \text { (by theorem 3) } \\
= & \cos \left(5^{n} \pi+\frac{2 \pi \cdot 5^{n}}{8 N}\right) \\
= & \cos \left(\pi+\frac{2 \pi \cdot 5^{n}}{8 N}\right) \\
= & -\cos \left(\frac{2 \pi \cdot 5^{n}}{8 N}\right)
\end{aligned}
$$

\section{References}

[1] N. Ahmed, T. Natarajan, and K. R. Rao, "Discrete cosine transform," IEEE Trans. Comput., vol.C-23, pp.89-93, Jan. 1974.

[2] M. J. Narasimha, and A. M. Peterson, "On the computation of the discrete cosine transform, "IEEE Trans. , COM-26, pp.934-946, June 1978.

[3] Z. Wang,"Fast algorithms for the discrete $W$ transform and for the discrtet Fourier transform, IEEE Trans, ASSP-32, pp.803-816, Aug. 1984.

[4] C. M. Rader, "Discrete Fourier transforms when the number of data samples is prime," Proc. IEEE, vol. 56, pp.1107-1108, June 1968.

[5] S. Winograd, "Some bilinear forms whóse multiplicative complexity depends on the field of constants," Math. Syst. Theory, 1977, vol.10, pp.169-180.

[6] K. H. Rosen, Elementary Number Theory and its Application, 1988.

[7] S. C. Pei and J. L. Wu,"Improved long convolutions using generalized number theoretic and polynomial transforms," IEEE Thans., ASSP-33, pp.1626-1627, Dec. 1985.

[8] M. Vetterli and H. J. Nussbaumer, "Simple FFT and DCT algorithms with reduced number of operations," Signal Processing, vol. 6, no.4, pp.267-278, Aug. 1984.

[9] H. S. Malvar, "Fast computation of the discrete cosine transform and the discrete Hartley transform," IEEE Trans., ASSP-35, pp.1484-1485, Oct. 1987. 